\title{
RIFAMPICIN ANTIBIOTIC IMPREGNATION OF THE ST. JUDE MEDICAL MECHANICAL VALVE SEWING RING: A WEAPON AGAINST ENDOCARDITIS
}

Bruce G. French, FRACS

Kathy Wilson, MVSc, BSc

Mae Wong, FRACPA

Susan Smith, BSc

Mark F. O'Brien, FRACS
The Dacron sewing ring material of the St. Jude Medical mechanical heart valve (St. Jude Medical, Inc., St. Paul, Minn.) was passively impregnated with rifampicin $(60 \mathrm{mg} / \mathrm{ml})$ both in its unsealed state and after sealing by the methods of preclotting in blood, autoclaving in blood, and autoclaving in $\mathbf{2 0 \%}$ albumin. Antistaphylococcal activity in the Dacron material was assessed immediately after rifampicin impregnation and at regular periods up to 5 days after implantation into the goat aorta. When the Dacron material had been sealed by autoclaving in blood and autoclaving in $\mathbf{2 0 \%}$ albumin significant retention of antistaphylococcal activity was found after 5 days in vivo. Best results were obtained with the use of autoclaved blood $(p<0.05)$. We also compared these results with those obtained from impregnating commercially available gelatin-sealed (Gelseal) and collagensealed (Hemashield) Dacron material with rifampicin. Although antistaphylococcal activity was equivalent immediately after rifampicin impregnation, after $\mathbf{4}$ days in vivo the activity was negligible in Gelseal material $(p<0.05)$ and could not be demonstrated in Hemashield material. Rifampicin impregnation of the intact St. Jude Medical mechanical valve sewing ring may have an application in the prevention of prosthetic valve endocarditis and a clinical protocol is suggested. (J Thorac Cardiovasc Surg 1996;112:248-52)
C ardiac valve replacement in the setting of active endocarditis carries a significant risk of persisting infection on the replacement device. ${ }^{1}$ Operation for aortic valve endocarditis is currently best managed with use of an aortic valve allograft. ${ }^{2}$ However, aortic valve allografts of the appropriate size are not always readily available in most cardiac surgical centers, especially in an emergency. In addition, patients with mitral valve endocarditis may not have conditions amenable to repair and consequently valve replacement with a prosthetic device is necessary. Therefore many patients with endocarditis undergo valve replacement with a mechanical or bioprosthetic valve. In such instances, prosthetic

From the Departments of Cardiac Surgery and Microbiology, The Prince Charles Hospital, Brisbane, Australia.

Received for publication April 20, 1995; revisions requested June 6, 1995; revisions received Oct. 20, 1995; accepted for publication Oct. 27, 1995.

Address for reprints: Mark O'Brien, FRCS, FRACS, Cardiac Surgeon-in-charge, Department of Cardiac Surgery, The Prince Charles Hospital, Rode Rd., Chermside, Queensland, Australia 4032.

Copyright (C) 1996 by Mosby-Year Book, Inc.

$0022-5223 / 96 \$ 5.00+0 \quad \mathbf{1 2 / 1 / 7 0 3 2 9}$ valve endocarditis (PVE), recurrent or persisting, will be an ever-present risk in the early postoperative phase. ${ }^{2}$

Bearing in mind that the site of infection in PVE is predominantly on the sewing ring, we postulated that a sewing ring resistant to early infection would significantly reduce the prevalence of PVE in patients at high risk. Previous studies by others have demonstrated that rifampicin can be passively incorporated into Dacron graft material. ${ }^{3-9}$ This ability has not been found with many other antibiotics. It has been shown that rifampicin can be ionically or covalently bound to an appropriate sealant such as human serum proteins, collagen, or gelatin that is bonded as a film to the Dacron graft material, which then acts as an antibiotic release system. For example, the N-4 piperazine group of rifampicin binds covalently to available carboxyl residues on gelatin in gelatin-sealed Dacron grafts. ${ }^{10}$ The retention of rifampicin activity in the prosthetic graft must then depend both on the affinity of the antibiotics for the graft sealant and on the rate of disappearance of the latter after implantation.

Sealing is a term that stems from the early role such treatments originally had in making the graft 
impervious to blood by coating the material with a film of protein.

This study attempts to evaluate the incorporation and retention of rifampicin into the Dacron sewing ring of the St. Jude Medical heart valve in an in vivo animal model.

\section{Material and methods}

The St. Jude Medical heart valve (St. Jude Medical, Inc., St Paul, Minn.) has a sewing ring constructed from unsealed double-velour knitted Dacron material. Eight millimeter dises of this material were made with a standard-sized punch. These discs were either left unsealed or sealed by one of the following methods: (1) preclotting with autologous blood, (2) autoclaving for 2 minutes with autologous blood, or (3) autoclaving for 2 minutes with $20 \%$ albumin. Rifampicin (Marion Merrell Dow, Pty. Ltd., Sydney, Australia) was made up to a solution of 60 $\mathrm{mg} / \mathrm{ml}$ in sterile $0.9 \% \mathrm{NaCl}$. The discs were then soaked in the rifampicin solution at room temperature for $15 \mathrm{~min}$ utes before goat intra-aortic implantation.

In addition, gelatin-sealed knitted Dacron material (Gelseal Triaxial, Vascutek, Renfrenshire, Scotland) and woven double-velour collagen-sealed Dacron material (Hemashield, Meadox Inc., Oakland, N.J.) were also used to compare the uptake and retention of rifampicin in these materials with that of the sewing ring material from the St. Jude Medical valve. Eight millimeter discs were cut and impregnated with rifampicin solution as described herein.

The antistaphylococcal activity in each disc was assessed (see microbiologic method section) according to the following design: (1) immediately after rifampicin impregnation (rifampicin uptake), (2) after 1 hour in vivo, (3) after 1 day in vivo, (4) after 2 days in vivo, (5) after 3 days in vivo, (6) after 4 days in vivo, and (7) after 5 days in vivo. Control discs sealed by the methods described but neither impregnated with rifampicin nor surgically implanted were also assessed for any inherent antistaphylococcal activity.

Animal study protocol. The in vivo component of the study was done in female Cashmere goats. A total of seven goats was used. Each goat was anesthetized with intravenous thiopental, intubated, and maintained on a $1.5 \%$ halothane/oxygen mixture. With use of sterile surgical technique and through a left thoracotomy the discs of rifampicin-impregnated Dacron material were secured to the luminal aspect of the descending thoracic aorta by a single horizontal mattress suture. Discs in triplicate, at least $1 \mathrm{~cm}$ apart, sealed by each method were implanted into the aorta at a different level through a separate aortotomy. The goat in which the rifampicin-impregnated discs were kept for 1 hour remained anesthetized with the chest open for 1 hour after release of the aortic crossclamps, whereupon it was killed with pentobarbital and the discs recovered. The other goats were all removed from the anesthetic and extubated. Intercostal nerve blocks with $0.5 \%$ bupivacaine were administered to each of these goats while the chest was being closed. All animals remained undistressed and made a good recovery from the thoracotomy. At the time intervals after implantation of 1 day, 2 days, 3 days, 4 days, and 5 days, the discs were recovered (after the goats were killed with pentobarbital) and assessed for antistaphylococcal activity. A single goat was the vehicle for all the discs for each period with one exception: two goats were used to carry discs for 4 days because our acquisition of the commercially available collagen-sealed Dacron material occurred after the other methods had already been assessed at 4 days.

Microbiologic method. Antistaphylococcal activity was assayed by placing the discs on an agar plate that had been inoculated for confluent growth of Staphylococcus aureus (strain ATCC25923, inoculum at $10^{7}$ colony-forming units per milliliter). The area of inhibition of growth around the disc at 24 hours was measured.

Tissue culture method. The effect of rifampicin on the tissue culture of fibroblasts from goat aorta and pericardium was also assessed. Before culture study, pieces of aortic or pericardial tissue were soaked in rifampicin solution $(60 \mathrm{mg} / \mathrm{ml}, 0.9 \% \mathrm{NaCl})$ at room temperature for 15 minutes. Chopped tissue pieces were cultured in medium 199 plus $10 \%$ fetal calf serum (CSL Ltd., Victoria, Australia) in $5 \% \mathrm{CO}^{2}$ at $37^{\circ} \mathrm{C}$ for 3 weeks. The subsequent outgrowth of fibroblasts was compared with that from untreated tissue.

Care of experimental animals. All animals received humane care in compliance with the principles of animal care as formulated by The National Health and Medical Research Council (Australia). This experimental study was approved by The Prince Charles Hospital Animal Ethics Committee (approval AEEC0693-02).

Statistical methods. Antistaphylococcal activity was measured as the mean area (in square centimeters) of growth inhibition on the agar plates from triplicate discs. The error was expressed as the range observed, with an accuracy in measuring the diameters of $\pm 1 \mathrm{~mm}$. Nonparametric statistical methods were used to analyze the data. The Kruskal-Wallis test was used to establish whether there was a difference between methods at all times. The Mann-Whitney $U$ test was used to compare specific methods. The level of significance used was $\alpha=0.05$.

\section{Results}

No antistaphylococcal activity was demonstrated in the control discs not impregnated with rifampicin, regardless of the sealant present.

Table I shows the rifampicin uptake, as expressed by antistaphylococcal activities at time 0 , for each of the methods used to seal the Dacron discs. The Kruskal-Wallis test verified a significant difference in the methods used (including and excluding the control discs). The highest levels of antistaphylococcal activity were observed in Dacron material sealed with autoclaved blood and autoclaved albumin. The Mann-Whitney U test was not able to identify one method as being consistently significantly better than the others; however, a subgrouping of Dacron discs sealed with autoclaved blood and autoclaved 


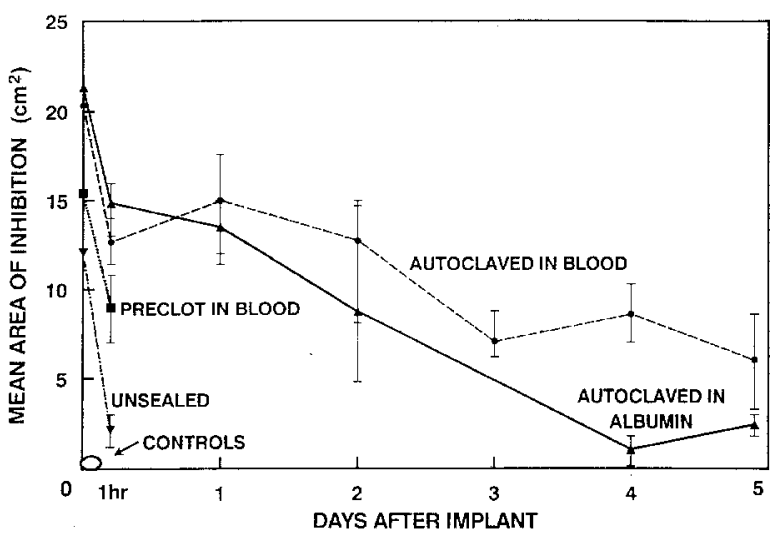

Fig. 1. Loss of rifampicin from Dacron discs measured as antistaphylococcal activity (inhibition of growth) for three methods of sealing Dacron sewing ring material and for unsealed Dacron material. Value for control discs is also plotted.

albumin versus a subgrouping of all the other methods demonstrated a highly significant difference $(p=0.0056$, Table I).

The loss of rifampicin antistaphylococcal activity from the discs over 5 days in vivo is shown in Figs. 1 and 2. Unsealed Dacron material impregnated with rifampicin lost most of its initial activity after only 1 hour in vivo. Similarly, for Dacron material sealed with clotted blood, there was a substantial loss of antistaphylococcal activity after 1 hour in vivo, despite the initial moderately high level of activity demonstrated before implantation. Dacron material sealed with autoclaved blood, however, still demonstrated a moderate level of antistaphylococcal activity after 5 days in vivo. Statistically equivalent antistaphylococcal activity was seen for up to 2 days between Dacron discs sealed with autoclaved blood and autoclaved 20\% albumin; however, by day 4 the former method proved to retain a significantly higher level of activity. Nevertheless, activity could still be demonstrated with Dacron material sealed with autoclaved albumin after 5 days in vivo (Fig. 1).

The antistaphylococcal activity in gelatin-sealed Dacron discs had fallen to almost negligible levels after 4 days; however, this was significantly higher than activity in Dacron discs sealed with collagen, which could not be demonstrated at 4 and 5 days after implantation (Fig. 2).

The difference demonstrated at time 0 between the two subgroups continued to be significant at all subsequent times.

Large numbers of fibroblasts were able to be cultured from both goat aorta and pericardium.
Table I. Rifampicin uptake

\begin{tabular}{lcc}
\hline \multicolumn{1}{c}{$\begin{array}{c}\text { Sealing } \\
\text { method }\end{array}$} & $\begin{array}{c}\text { Inhibition: } \text { Mean } \\
\text { area }\left(\mathrm{cm}^{2}\right) \text { (range) }\end{array}$ & Subgroup \\
\hline Autoclaved in blood & $20.34(14.69-24.11)$ & 1 \\
Autoclaved in 20\% albumin & $21.34(18.34-24.11)$ & 1 \\
Gelatin sealed & $18.95(16.84-21.65)$ & 2 \\
Preclotted in blood & $15.40(14.70-16.11)$ & 2 \\
Collagen sealed & $15.02(11.44-18.34)$ & 2 \\
Unsealed & $12.06(11.44-12.70)$ & 2 \\
Control & 0 & 0 \\
\hline
\end{tabular}

Uptake of rifampicin by the Dacron sewing ring material measured as antistaphylococcal activity (inhibition of growth) at time 0 for unsealed Dacron material, the five sealant methods, and the control discs (not rifampicin soaked).

Although growth was not assayed quantitatively, rifampicin did greatly diminish the outgrowth of fibroblasts from the rifampicin-soaked tissue pieces.

\section{Discussion}

PVE is an important complication of prosthetic valve replacement for endocarditis. It has recently been reported that the 1-year freedom from PVE in this setting is $87 \% .^{11}$ Infection predominantly affects the host tissues attaching to the sewing ring of the prosthesis, although in bioprosthetic valves it can affect the leaflet tissue. The ability to retain local antibacterial activity in the sewing ring of a prosthetic valve might therefore decrease the risk of PVE in the period immediately after valve implantation.

Powell, Burnham, and Johnson ${ }^{3}$ added rifampicin to the blood used for preclotting the Dacron material rather than soaking the clotted graft as we have done. This resulted in good retention 24 hours after implantation into the dog. Other workers have attempted to duplicate these experiments by using commercially available sealed Dacron fabric arterial grafts and simulating in vivo conditions by agitating the rifampicin-loaded Dacron material in an albu$\mathrm{min} / \mathrm{saline}$ solution mixture. ${ }^{7,8}$ With these techniques Chervu and associates ${ }^{7}$ found that rifampicin-impregnated collagen-sealed grafts retained antistaphylococcal activity for 22.4 days, which was significantly better than the duration of antistaphylococcal activity in rifampicin-impregnated preclotted grafts. This result was not supported in a study by Ashton and colleagues, ${ }^{8}$ who found that rifampicin activity in collagen-sealed grafts could only be demonstrated for 1 day of simulated in vivo conditions, but with gelatin-sealed grafts the activity could be demonstrated for up to 4 days.

Our results were obtained with the use of true in 
vivo conditions. They do support the finding that rifampicin appears to be better retained in gelatinsealed Dacron material than in collagen-sealed Dacron material, although we recognize that our study did not fully assess these grafts in the initial few days of the in vivo period. More important, however, we found that better retention occurs when rifampicin is added to Dacron material that has first been sealed by autoclaving in the animal's blood and, to a somewhat lesser extent, by autoclaving in $20 \%$ albumin. In addition, our results show that the autoclaved blood technique yields significantly better antistaphylococcal activity after 4 and 5 days in vivo than the autoclaved $20 \%$ albumin technique.

One problem encountered with the Dacron discs sealed by autoclaving in blood was an increased resistance of the material to needle penetration. This may be of some concern, inasmuch as the unsealed St. Jude Medical valve sewing ring by itself does not permit easy needle passage. Neither autoclaving in $20 \%$ albumin nor the presence of gelatin or collagen appears to affect the handling characteristics of the Dacron material.

We would point out that the techniques described of sealing Dacron material by autoclaving in blood or albumin, when applied to the intact valve, carry a risk of causing particulate matter to adhere to the valve leaflets, which may interfere with leaflet function or could possibly affect the prevalence of valve thrombosis. This problem can be overcome by ensuring that no blood remains on the valve leaflets before autoclaving and that the leaflets are meticulously cleaned of all particulate matter after autoclaving.

The unfavorable effect of rifampicin on fibroblast culture found in this study is difficult to evaluate. Because it is likely that endothelial cells would be at least as sensitive as fibroblasts, it is possible to postulate that the high concentrations of rifampicin in the sewing ring of an implanted, impregnated valve may impair or delay initial endothelialization if such occurs in the first several days. The clinical significance of this is questionable because fibroblastic penetration into parts of the sewing ring may be more important and this does not begin to take place for several days. In practice the effect may be negligible; however, it is beyond the scope of this study to address whether this would cause an increase in the prevalence of periprosthetic leakage caused by the early temporary inhibition of endothelialization.

This study has shown that rifampicin can be satisfactorily incorporated into the sewing ring ma-

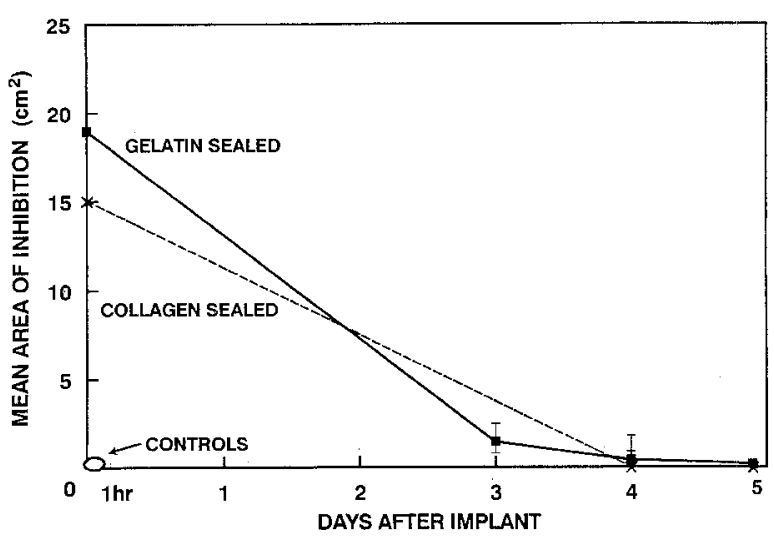

Fig. 2. Loss of rifampicin from commercially available gelatin-sealed and collagen-sealed Dacron material measured as antistaphylococcal activity (inhibition of growth).

terial of the St. Jude Medical heart valve and that by first autoclaving the material in blood, antistaphylococcal activity is retained for at least 5 days in the experimental animal. Rifampicin impregnation of sewing ring material after autoclaving in $20 \%$ albumin yields similar antistaphylococcal activity for 2 days in vivo but significantly less activity by 4 days. Both of these methods of impregnating rifampicin into the sewing ring material of the St. Jude Medical heart valve allowed significantly better retention of antistaphylococcal activity than observed with either rifampicin-impregnated gelatin-sealed or collagensealed Dacron material.

Ideally a prospective clinical trial should be done to determine the beneficial effects and any possible detrimental effects of this procedure, which may be based on the following suggested clinical application.

Possible clinical application and suggested protocol. The patient is one with endocarditis who requires valve replacement with a prosthetic valve. After the appropriate-sized mechanical valve is selected, it is soaked in heparinized autologous blood and then autoclaved for 2 minutes. The valve is carefully cleaned of all particulate matter and immersed in the rifampicin solution at room temperature for 15 minutes. This solution is composed of rifampicin in $0.9 \% \mathrm{NaCl}$ made up to a concentration of $60 \mathrm{mg} / \mathrm{ml}$. During this waiting period multiple interrupted polypropylene sutures can be placed in the anulus in the usual manner. Polypropylene rather than braided suture material is used because it is less liable to become a nidus for residual infection. The rifampicin-impregnated valve is then 
inserted. Appropriate systemic administration of antibiotics is continued according to the nature and extent of the endocarditis.

If passage of the needle through the Dacron fabric sewing rim is considered too difficult, acceptable antistaphylococcal activity can still be obtained by soaking the valve in $20 \%$ albumin instead of autologous blood before autoclaving the valve. This activity is as good initially but diminishes over a shorter interval.

This protocol is also eminently suitable for Dacron fabric tube arterial grafts in the presence of surrounding infection. Both experimental and clinical experiences with arterial grafts have been reported, $, 6,7,10,12-14$ and the use of antibiotic-soaked grafts in vascular operations is reasonably well established.

We express appreciation for the kind donation by St. Jude Medical, Inc., of the sewing ring material and the Hemashield collagen-sealed graft material; to Vascutek for the donation of the Gelseal Triaxial graft material, and to Marion Merrell Dow for the donation of the rifampicin. The following Prince Charles Hospital staff are also thanked for their technical assistance: Bill Sommers (Animal House Attendant) and Christine Willmette (Heart Valve Technician) for assistance with the surgical procedures, Ken Gall (Heart Valve Coordinator) for setup and maintenance of the fibroblast cultures, and Paul Hampshire (Clinical Microbiologist) for assay of the antistaphylococcal activity.

\section{REFERENCES}

1. Ivert TSA, Dismukes WE, Cobbs CG, Blackstone EH, Kirklin JW, Bergdahl LA. Prosthetic valve endocarditis. Circulation 1984;69:223-32.

2. McGiffin DC, Galbraith AJ, McLachlan GJ, et al. Aortic valve infection: risk factors for death and recurrent endocarditis after aortic valve replacement. J Thorac Cardiovasc Surg 1992;104:511-20.
3. Powell TW, Burnham SJ; Johnson G. A passive system using rifampicin to create an infection-resistant vascular prosthesis. Surgery 1983;94:765-8.

4. Avramovic JR, Fletcher JP. Rifampicin impregnation of a protein-sealed Dacron graft: an infection-resistant prosthetic vascular graft. Aust N Z J Surg 1991;61:436-40.

5. Colburn MD, Moore WS, Chvapil M, Gelabert HA, Quinones-Baldrich WJ. Use of an antibiotic-bonded graft for in situ reconstruction after prosthetic graft infections. J Vasc Surg 1992;16:651-60.

6. Torsello G, Sandmann W, Gehrt A, Jungblut RM. In situ replacement of infected vascular prostheses with rifampicinsoaked vascular grafts: early results. J Vasc Surg 1993;17:76873.

7. Chervu A, Moore WS, Chvapil M, Henderson T. Efficacy and duration of antistaphylococcal activity comparing three antibiotics bonded to Dacron vascular grafts with a collagen release system. J Vasc Surg 1991;13:897-901.

8. Ashton TR, Cunningham D, Paton D, Maini R. Antibiotic loading of vascular grafts [Abstract]. Proc Soc Biomat 1990; 13:235.

9. Goeau-Brissoniere O, Mercier F, Nicolas MH, et al. Treatment of vascular graft infection by in situ replacement with a rifampicin-bonded gelatin-sealed Dacron graft. J Vasc Surg 1994;19:739-44.

10. Strachan CJL, Newsom SWB, Ashton TR. The clinical use of an antibiotic-bonded graft. Eur J Vasc Surg 1991;5:627-32.

11. Haydoc D, Barratt-Boyes B, Macedo T, Kirklin JW, Blackstone $\mathrm{E}$. Aortic valve replacement for active infectious endocarditis in 108 patients: a comparison of freehand allograft valves with mechanical prostheses and bioprostheses. J Thorac Cardiovasc Surg 1992;103:130-9.

12. Lachapelle K, Graham AM, Symes JF. Antibacterial activity, antibiotic retention and infection resistance of a rifampinimpregnated gelatin-sealed Dacron graft. J Vasc Surg 1994; 19:675-82.

13. Avramovic J, Fletcher JP. Prevention of prosthetic vascular graft infection by rifampicin impregnation of a protein-sealed Dacron graft in combination with parental cephalosporin. J Cardiovasc Surg 1992;33:70-4.

14. Freyrie $\mathrm{A}$, Curti $\mathrm{T}$, Rodio $\mathrm{M}$, et al. Infection between vascular prostheses and rifampicin in the prevention of the graft's infection. Int Angiol 1992;11:113-6. 